\title{
CONTROLANDO A GRIPE SUÍNA VIA VACINAÇÃO: UM ESTUDO EPIDEMIOLÓGICO BASEADO EM AUTÔMATO CELULAR E EQUAÇÕES DIFERENCIAIS
}

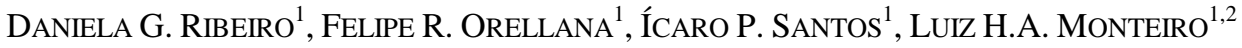 \\ 1. Escola de Engenharia da Universidade Presbiteriana Mackenzie
}

2. Escola Politécnica da Universidade de São Paulo

E-mails: luizm@mackenzie.br; luizm@usp.br

\begin{abstract}
Here the effects of two vaccination strategies on the propagation of contagious diseases are evaluated by using an epidemic model based on probabilistic cellular automaton. The mean-field approximation written in terms of ordinary differential equations is also analyzed and used in this study. As an example, the performance of these two vaccination strategies against the spreading of the swine influenza virus is numerically investigated.
\end{abstract}

Keywords — bifurcation, epidemiology, population dynamics, probabilistic cellular automaton, vaccination.

Resumo - Aqui, avaliam-se os efeitos de duas estratégias de vacinação sobre a propagação de doenças contagiosas, usando um modelo epidemiológico baseado em autômato celular probabilista. A aproximação de campo-médio escrita em termos de equações diferenciais ordinárias é também analisada e usada neste estudo. Como exemplo, investiga-se, numericamente, o desempenho dessas duas estratégias de vacinação contra a disseminação do vírus da gripe suína.

Palavras-chave — autômato celular probabilista, bifurcação, dinâmica de populações, epidemiologia, vacinação.

\section{Introdução}

Doenças contagiosas preocupam as pessoas e os governos. Exemplos clássicos de epidemias são a peste negra, que dizimou cerca de $30 \%$ da população europeia no século XIV, e a gripe espanhola, que matou entre 1918 e 1919 mais de 100 milhões de pessoas no mundo (Anderson e May, 1992; Murray, 2002; Murray, 2003). Um exemplo talvez menos conhecido, mas de gravidade equivalente, é o da malária, que mata anualmente mais de um milhão de africanos (WHO, 2012).

Felizmente, a propagação de algumas doenças contagiosas pode ser controlada por meio de vacinação. Usualmente, vacinas são aplicadas na população hospedeira de duas maneiras: ou continuamente (ou seja, diariamente, uma pequena quantidade de indivíduos é vacinada) ou periodicamente (de modo que muitos indivíduos são vacinados num intervalo de tempo curto, de tempos em tempos). A primeira estratégia é chamada de vacinação constante; a segunda, de vacinação pulsada (Shulgin et al., 1998). Por exemplo, no Brasil, crianças devem tomar vacina contra caxumba, rubéola e sarampo ao completarem 12 meses de vida (Ministério da Saúde, 2010); portanto, uma pequena parcela da população é vacinada diariamente contra essas doenças. Já pessoas com mais de 60 anos devem tomar vacina contra gripe anualmente, geralmente, entre o final do mês de abril e o início do mês de maio (Ministério da Saúde, 2013). O primeiro caso é um exemplo de vacinação constante; o segundo, de vacinação pulsada.
Aqui, usa-se um modelo epidemiológico do tipo SIR (suscetível-infectado-recuperado) (Anderson e May, 1992; Murray, 2002), representado por meio de um autômato celular probabilista (ACP), a fim de avaliar os efeitos dessas duas estratégias de vacinação. A aproximação de campo médio correspondente, escrita em termos de equações diferenciais ordinárias (EDO), é também analisada e utilizada neste estudo.

\section{Propósito}

Neste artigo, investiga-se, numericamente, o desempenho de dois esquemas de vacinação usados para controlar a propagação do vírus $\mathrm{A} / \mathrm{H} 1 \mathrm{~N} 1$, causador da gripe suína. Recentemente, modelos baseados em autômatos celulares têm sido usados para analisar diferentes aspectos da infecção por H1N1. Por exemplo, Qian et al. (2011) concluíram que é crucial isolar os indivíduos contaminados a fim de conter a propagação desse vírus; Lee et al. (2011) e Prosser et al. (2011) avaliaram a relação custo-benefício da vacinação contra tal vírus e afirmaram que é importante facilitar o acesso à vacina a todas as faixas etárias.

$\mathrm{Na}$ próxima seção, descrevem-se os modelos formulados em termos de ACP e EDO, e a patologia causada pelo $\mathrm{H} 1 \mathrm{~N} 1$.

\section{Os modelos e a doença}

ACP tem sido usado na modelagem da propagação de doenças contagiosas (Monteiro et al., 2006; Schimit e Monteiro, 2009). No nosso modelo, cada indivíduo da população hospedeira ocupa uma célula 
do ACP, cujo reticulado corresponde a uma matriz de dimensões $n \times n$ com condições de contorno periódicas (ou seja, a primeira linha do reticulado está em contato com a última linha e a primeira coluna está em contato com a última coluna; assim, os indivíduos vivem numa superfície toroidal, de modo que não há efeitos de borda). Cada célula está em contato com as oito ao seu redor, o que no jargão de autômatos celulares é chamado de vizinhança de Moore de raio unitário (Wolfram, 1994).

Cada célula (cada indivíduo) está, a cada passo de tempo $t$, em um de três estados: suscetível $(S)$, infectado $(I)$ ou recuperado $(R)$. Por simplicidade, assume-se que o número de indivíduos da população hospedeira permanece constante durante o intervalo de tempo em que a propagação da doença é investigada. Assim, quando um indivíduo $S, I$ ou $R$ morre, nasce um indivíduo $S$ em seu lugar. Essa suposição é razoável no caso em que a taxa de natalidade da população é próxima da taxa de mortalidade.

A propagação de doença contagiosa é aqui modelada pelas regras probabilistas descritas a seguir. Tais regras governam a transição de estados do ACP.

Assume-se que, a cada passo de tempo $t$, há uma probabilidade $P_{\text {inf }}$ de um suscetível se contaminar e se tornar infectado, dada por $P_{\text {inf }}=1-e^{-K v}$, sendo $v$ o número de vizinhos doentes e $K$ o parâmetro que expressa a infectividade da doença (a facilidade com que o agente patogênico é transmitido e se instala num suscetível). Essa regra descreve a transição $S \rightarrow I$. Observe que o valor de $P_{\text {inf }}$ cresce com o aumento de $v$ e/ou de $K$, e que se $v=0$, então $P_{\text {inf }}=0$.

A cada instante $t$, cada infectado pode se curar com probabilidade $P_{\text {cura }}$, o que corresponde a uma transição $I \rightarrow R$; se não se curou, ele pode morrer devido à doença com probabilidade $P_{\text {morte-1 }}$, o que corresponde a uma transição $I \rightarrow S$; se não se curou nem morreu, ele permanece infectado. Supõe-se que a cura leva à imunidade plena.

Ainda, a cada passo de tempo $t$, cada recuperado pode morrer por causas não relacionadas à doença com probabilidade $P_{\text {morte-2. }}$. Quando isso ocorre, temse uma transição $R \rightarrow S$. Essa probabilidade também engloba o processo de perda de imunidade, de modo que um recuperado volta, espontaneamente, a se tornar suscetível ao patógeno em estudo.

Supõe-se também que, a cada instante $t$, um suscetível pode ser vacinado com probabilidade $P_{v a c}$ e que a vacina confere imunidade plena. Essa regra está associada à transição $S \rightarrow R$.

Em termos de equações diferenciais ordinárias, esse ACP escreve-se assim:

$$
\begin{aligned}
& d S(t) / d t=-a S(t) I(t)+c I(t)+e R(t)-f S(t) \\
& d I(t) / d t=a S(t) I(t)-b I(t)-c I(t) \\
& d R(t) / d t=b I(t)-e R(t)+f S(t)
\end{aligned}
$$

sendo $S(t), I(t)$ e $R(t)$ as quantidades de indivíduos suscetíveis, infectados e recuperados, respectivamente. Note que como $d S(t) / d t+d I(t) / d t+d R(t) / d t=0$, então $S(t)+I(t)+R(t)=N=$ constante, sendo $N=n^{2}$ o número total de indivíduos da população.
A equivalência entre o ACP e sua aproximação de campo-médio escrita em termos de EDO se dá tomando (Monteiro et al., 2006; Schimit e Monteiro, 2009): $b=P_{\text {cura }}, c=\left(1-P_{\text {cura }}\right) P_{\text {morte- } 1}, e=P_{\text {morte- } 2}, f=P_{\text {vac }} \mathrm{e}$ $a=\Delta_{S \rightarrow I}\{S(t) I(t)]$, com $\Delta_{S \rightarrow I}$ denotando o número de transições $S \rightarrow I$ ocorridas por passo de tempo, após o sistema atingir seu regime permanente.

O sistema (1)-(3) possui duas soluções estacionárias (soluções tais que $d S(t) / d t=0, d I(t) / d t=0 \mathrm{e}$ $d R(t) / d t=0)$, dadas por: $\left(S_{1}{ }^{*}, I_{1}{ }^{*}\right)=(e N /(e+f), 0)$ e $\left.\left(S_{2}{ }^{*}, I_{2}{ }^{*}\right)=\left((b+c) / a, e N\left(R_{0}-1\right) /(b+e) R_{0}\right]\right)$, com $R_{0} \equiv$ $a e N /(b+c)(e+f)]$. A primeira solução é chamada de livre-de-doença (pois $I_{1}{ }^{*}=0$ ); a segunda, de solução endêmica (pois $I_{2}{ }^{*}>0$ ). Obviamente, nos dois casos, $R^{*}=N-\left(S^{*}+I^{*}\right)$.

A estabilidade dessas soluções de equilíbrio pode ser inferida a partir dos autovalores da matriz jacobiana, obtida linearizando o sistema (1)-(3) em torno de tais soluções. De acordo com o teorema de Hartman-Grobman, $\left(S^{*}, I^{*}\right)$ é assintoticamente estável se seus autovalores correspondentes têm parte real negativa. Mostra-se, para o sistema em análise, que a solução livre-de-doença é assintoticamente estável para $R_{0}<1$ e instável para $R_{0}>1$, e que a solução endêmica é assintoticamente estável para $R_{0}>1 \mathrm{e}$ instável para $R_{0}<1$. Portanto, em $R_{0}=1$, ocorre uma bifurcação transcrítica (Monteiro, 2011). Essa bifurcação acontece quando duas soluções de equilíbrio trocam de estabilidade conforme um parâmetro do sistema passa por um valor crítico: assim, aquela solução que era assintoticamente estável passa a ser instável, e a que era instável se torna assintoticamente estável.

O parâmetro de bifurcação $R_{0}$ é, no jargão de epidemiologia, chamado de número (ou fator) de reprodutividade basal (Anderson e May, 1991; Murray, 2002). Tal parâmetro reflete o número médio de infecções secundárias causadas por um único indivíduo doente inserido numa população suscetível. Por isso, quando $R_{0}>1$, o patógeno correspondente invade e/ou persiste na população; quando $R_{0}<1$, ele tende a desaparecer.

O modelo epidemiológico aqui proposto pode representar a evolução temporal das quantidades de indivíduos $S, I$ e $R$ no caso de doenças contagiosas contra as quais há vacina. Um exemplo é a gripe; em particular, a gripe suína, uma doença viral causada pelo subtipo A/H1N1. Sua taxa de mortalidade é de 1-10 para cada 10.000 infectados e $R_{0} \approx 1,5-3,5$ (isto é, cada infectado tende a contaminar, na média, de 1,5 a 3,5 suscetíveis). Em geral, o doente se cura naturalmente após alguns dias (Ministério da Saúde, 2010; Modchang et al., 2012; Nishiura et al., 2010).

O Ministério da Saúde do Brasil aplica, gratuitamente, vacina contra gripe nas pessoas pertencentes aos grupos de maior risco de sofrerem complicações em consequência de contraírem essa doença. Esses grupos prioritários são: crianças com 6 a 24 meses de vida, idosos acima de 60 anos, indígenas, gestantes, mulheres que deram à luz nos últimos 45 dias, pessoas com doenças crônicas (como asma ou 
diabetes), presidiários, profissionais da área da saúde que lidam com possíveis infectados. Em 2013, o governo disponibilizou 43 milhões de doses de vacina contra os subtipos mais comuns do vírus encontrados no ano passado, que são o $\mathrm{A} / \mathrm{H} 1 \mathrm{~N} 1$, o A/H3N2 e o B. Sua meta era vacinar ao menos $80 \%$ do público-alvo (Ministério da Saúde, 2013).

A seguir, apresentam-se os resultados obtidos a partir de simulações numéricas com o ACP, usando valores de parâmetros típicos da gripe suína.

\section{Resultados}

Considere que um passo de tempo do ACP corresponda a 3 dias de tempo real e que $n=200$ (portanto, há 40.000 indivíduos na região espacial representada pelo reticulado do ACP). Inicialmente, assuma que $P_{\text {vac }}=0$ (ou seja, não há vacinação); $P_{\text {cura }}=0,7$ (assim, na média, 70\% dos infectados se curam em 3 dias, $(1-0,7) \times 0,7+0,7=91 \%$ se curam em 6 dias, etc.); $P_{\text {morte- }}=0,0005$ (compatível com a taxa de mortalidade da doença, que é de 1 a 10 para 10.000 infectados); $P_{\text {morte-2 }}=0,1$ (desse modo, na média, $10 \%$ dos recuperados ou morrem ou perdem a imunidade a cada passo de tempo) e $K=1$. Com essas escolhas, estimando o valor do parâmetro $a$ e dos demais parâmetros das equações (1)-(3) conforme descrito na seção anterior, obtêm-se, via simulações numéricas, $a N=1,67 \pm 0,02$ e $R_{0}=2,38 \pm 0,01$. Essas médias e os respectivos desvios padrões foram calculados a partir de 10 simulações, cada uma com 200 passos de tempo. Note que o valor de $R_{0}$ encontrado é maior do que um (portanto, o patógeno se torna endêmico) e é compatível com o da doença modelada. A condição inicial das simulações é $S(0) / N=0,99, I(0) / N=0,01$ e $R(0) / N=0$; isto é, $99 \%$ da população é suscetível e $1 \%$ está infectada em $t=0$. A solução estacionária atingida (endêmica) é composta por $42 \%$ de suscetíveis, $7 \%$ de infectados e $51 \%$ de recuperados, o que está de acordo com os valores calculados a partir das expressões para $S_{2}{ }^{*}$ e $I_{2}{ }^{*}$. A figura 1 ilustra uma dessas 10 simulações.

O objetivo deste trabalho é avaliar o efeito de duas estratégias de vacinação sobre a propagação da doença contagiosa em análise. Para isso, considere que o tempo total de simulação seja 120 passos de tempo (o que equivale a 1 ano de tempo real). Nesse intervalo, as simulações mostram que, para $n=200$, a doença mata $53 \pm 6$ indivíduos, se $P_{v a c}=0$.

Considere, agora, um valor não nulo para $P_{v a c}$; por exemplo, $P_{v a c}=0,05$. Nesse caso, obtém-se $R_{0}=$ $1,27 \pm 0,02$ (um valor mais próximo do número crítico $R_{0}=1$ ); além disso, a doença mata $17 \pm 3$ indivíduos (menos do que no caso sem vacinação, como esperado) e são aplicadas $(1,34 \pm 0,02) \times 10^{5}$ doses de vacina, no intervalo de tempo mencionado. Note que essa quantidade de doses é cerca de três vezes maior do que a população total, que é de $4 \times 10^{4}$ indivíduos. Portanto, o esquema de vacinação em que, a cada passo de tempo, há a probabilidade $P_{v a c}$ de cada sus- cetível ser vacinado, não parece ser eficiente, pois se podem gastar mais vacinas do que a população total e, mesmo assim, a doença se tornar endêmica. Numericamente, verifica-se que a doença tende a ser erradicada para $P_{v a c} \geq 0,07$. Por exemplo, para $P_{v a c}=0,07$, gastam-se $(1,873 \pm 0,007) \times 10^{5}$ vacinas na erradicação e, ainda assim, ocorrem $8 \pm 2$ mortes. A figura 2 exibe um exemplo de erradicação usando vacinação constante.

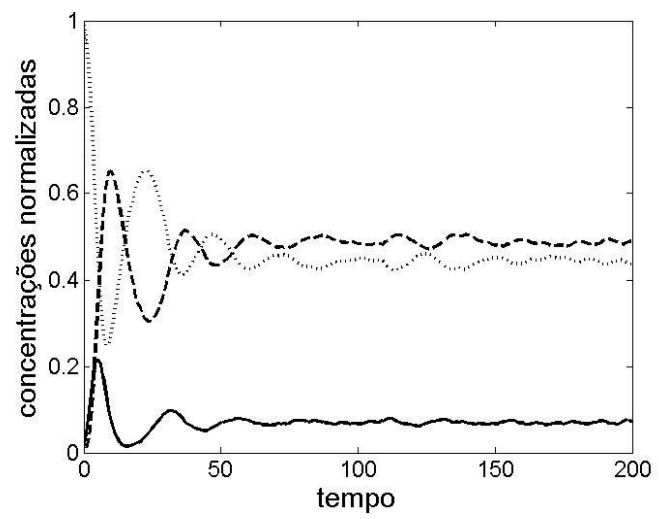

Figura 1. Evoluções temporais de $S(t) / N$ (linha pontilhada), $I(t) / N$ (linha cheia) e $R(t) / N$ (linha tracejada) para $K=1, P_{\text {cura }}=70 \%$, $P_{\text {morte }-1}=0,05 \%, P_{\text {morte }-2}=10 \%, P_{\text {vac }}=0$ e $n=200$, a partir da condição inicial $S(0) / N=99 \%, I(0) / N=1 \%$ e $R(0) / N=0$. Note que a doença se torna endêmica na população.

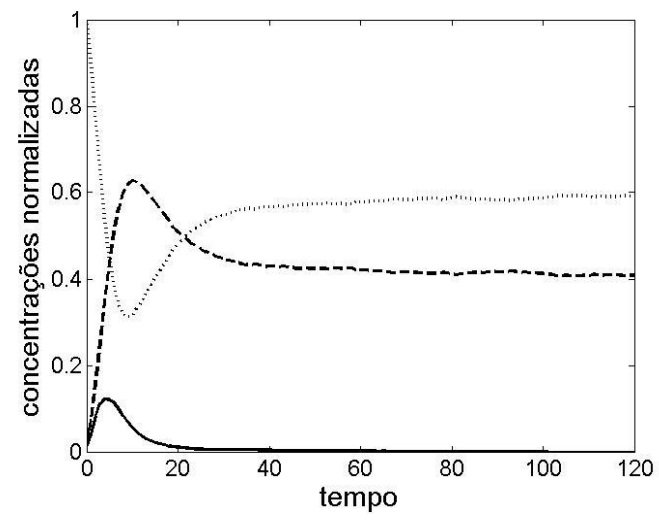

Figura 2. Evoluções temporais de $S(t) / N$ (linha pontilhada), $I(t) / N$ (linha cheia) e $R(t) / N$ (linha tracejada) para $K=1, P_{\text {cura }}=70 \%$, $P_{\text {morte- }}=0,05 \%, P_{\text {morte } 2}=10 \%, P_{v a c}=7 \%$ e $n=200$, a partir da condição inicial $S(0) / N=99 \%, I(0) / N=1 \%$ e $R(0) / N=0$. Após 120 passos de tempo, a doença está eliminada.

Vacinação pulsada é representada, no nosso modelo, escolhendo uma quantidade inicial de recuperados não nula. Desse modo, o valor de $R(0) / N>0$ expressa a fração da população que tomou a vacina e ficou imune à doença. De acordo com esse esquema, para $I(0) / N=1 \%$ (como anteriormente considerado), as simulações numéricas mostram que a doença não é erradicada nem se $R(0) / N=99 \%$; ou seja, nem vacinando simultaneamente toda a população não infectada, como mostra a figura 3. Com esse esquema só se obtém erradicação vacinando ao menos 99,9\% (nesse caso, a fração inicial de infectados deve ser, no máximo, de $0,1 \%$ ). Isso corresponde a gastar cerca de $4 \times 10^{4}$ vacinas; ou seja, praticamente toda a população deve ser vacinada. 


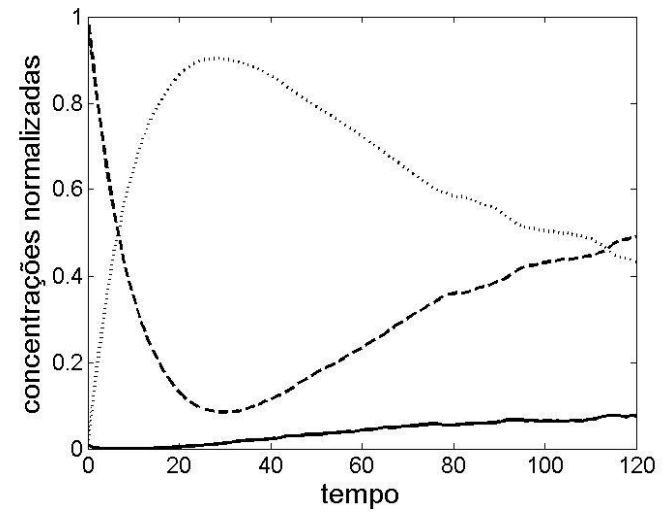

Figura 3. Evoluções temporais de $S(t) / N$ (linha pontilhada), $I(t) / N$ (linha cheia) e $R(t) / N$ (linha tracejada) para $K=1, P_{\text {cura }}=70 \%$, $P_{\text {morte- } 1}=0,05 \%, P_{\text {morte }-2}=10 \%, P_{\text {vac }}=0$ e $n=200$, a partir da condição inicial $S(0) / N=0 \%, I(0) / N=1 \%$ e $R(0) / N=99 \%$. As concentrações tendem aos valores estacionários mostrados da figura 1.

\section{Discussão e conclusão}

Erradicar o vírus da gripe não parece ser uma preocupação dos governos ao redor do mundo. Em geral, o que eles parecem almejar é controlar sua propagação, buscando minimizar o número de óbitos das pessoas mais sujeitas a complicações decorrentes da infecção. No Brasil, não há vacina gratuita para todos; há apenas para aqueles pertencentes aos grupos de risco.

De acordo com as simulações realizadas, vacinação pulsada deu melhores resultados do que vacinação constante, pois pode levar à erradicação gastando menos doses de vacina. De fato, aplicando 39.960 doses num único instante $(99,9 \%$ de 40.000$)$ geralmente se obtém erradicação, enquanto que vacinação constante exige 187.300 doses, um número quase cinco vezes maior. Vacinação pulsada foi o esquema adotado pelas autoridades de saúde para erradicar a poliomielite do nosso país (Risi, 1984). Entretanto, vacinação constante foi usada mundialmente para erradicar a varíola (Behbehani, 1983). Ou seja, ambos os esquemas já foram usados com sucesso.

Para finalizar, é importante ressaltar que nosso modelo simplista não considera:

- a imigração de infectados para a região espacial analisada; isto é, não leva em conta que o vírus pode ser reintroduzido na população hospedeira;

- a possibilidade de surgir uma variação do vírus, via mutação genética, que tornaria suscetível aqueles considerados imunes;

- a imunidade, adquirida após uma infecção, poder ser apenas parcial (em vez de plena);

- a variação sazonal do parâmetro de infectividade $K$ com as condições climáticas, já que, em geral, fica-se gripado mais facilmente no inverno do que no verão (Eccles, 2002).

Esses aspectos serão levados em conta futuramente.

\section{Agradecimento}

L.H.A. Monteiro agradece o apoio do CNPq.

\section{Referências bibliográficas}

Anderson R.M. e May R.M. (1991). Infectious Diseases of Humans. Oxford University Press, Oxford.

Behbehani A.M. (1983). The Smallpox Story: Life and Death of an Old Disease. Microbiological Reviews, Vol. 47, No. 4 pp. $455-509$

Eccles R. (2002). An Explanation for the Seasonality of Acute Upper Respiratory Tract Viral Infections. Acta Otolaryngo-logica, Vol. 122, No. 2, pp. 183-191. DOI: 10.1080/00016480252814207

Lee B.Y., Bacon K.M., Donohue J.M., Wiringa A.E., Bailey R.R. e Zimmerman R.K. (2011). From the Patient Perspective: The Economic Value of Seasonal and H1N1 Influenza Vaccination. Vaccine, Vol. 29, No. 11, pp. 2149-2158. DOI: 10.1016/j.vaccine.2010.12.078

Ministério da Saúde do Brasil (2010). Doenças Infecciosas e Parasitárias. Editora MS, Brasília.

Ministério da Saúde do Brasil (2013). Campanha Nacional de Vacinação contra a Influenza - Ano 2013. Em: http://portal.saude.gov.br/portal/arquivos/pdf/informe_tecnic o_campanha_influenza_2013_svs_pni.pdf

Modchang C., Iamsirithaworn S., Auewarakul P. e Triampo W. (2012). A Modeling Study of School Closure to Reduce Influenza Transmission: A Case Study of an Influenza A (H1N1) Outbreak in a Private Thai School. Mathematical and Computer Modelling, Vol. 55, No. 3-4, pp. 1021-1033. DOI: 10.1016/j.mcm.2011.09.027

Monteiro L.H.A. (2011). Sistemas Dinâmicos, $3^{\mathrm{a}}$ ed., Livraria da Física, São Paulo.

Monteiro L.H.A., Chimara H.D.B. e Berlinck J.G.C. (2006). Big Cities: Shelters for Contagious Diseases. Ecological Modelling, Vol. 197, No. 1-2, pp. 258-262. DOI: 10.1016/j.ecolmodel.2006.02.042

Murray J.D. (2002). Mathematical Biology I: Introduction. Springer, New York.

Murray J.D. (2003). Mathematical Biology II: Spatial Models and Biomedical Applications. Springer, New York.

Nishiura H., Chowell G., Safan M. e Castillo-Chavez C. (2010). Pros and Cons of Estimating the Reproduction Number from Early Epidemic Growth Rate of influenza A (H1N1) 2009. Theoretical Biology and Medical Modelling, Vol. 7, No. 1, artigo 1. DOI: 10.1186/1742-4682-7-1

Prosser L.A., Lavelle T.A., Fiore A.E., Bridges C.B., Reed C., Jain S., Dunhuam K.M. e Meltzer M.I. (2011). CostEffectiveness of 2009 Pandemic Influenza A (H1N1) Vaccination in the United States. PLoS ONE, Vol. 6, No. 7, artigo e22308. DOI: 10.1371/journal.pone.0022308

Qian Y., Ren Z., Lin F. e Zeng Q. (2011). How The Swine Lineage Influenza A (H1N1) Virus Spread? Key Engineering Materials, Vol. 460-416, pp. 21-25.

Risi Jr. J.B. (1984). The Control of Poliomyelitis in Brazil. Reviews of Infectious Diseases, Vol. 6, Suppl. 2, pp. S400-S403. DOI: 10.1093/clinids/6.Supplement_2.S400

Schimit P.H.T. e Monteiro L.H.A. (2009). On the Basic Reproduction Number and the Topological Properties of the Contact Network: An Epidemiological Study in Mainly Locally Connected Cellular Automata. Ecological Modelling, Vol. 220, No. 7, pp. 1034-1042. DOI: 10.1016/j.ecolmodel.2009.01.014

Shulgin B., Stone L. e Agur Z. (1998). Pulse Vaccination Strategy in the SIR Epidemic Model. Bulletin of Mathematical Biology, Vol. 60, No. 6, pp. 1123-1148. DOI: 10.1016/S0092-8240(98)90005-2

Wolfram S. (1994). Cellular Automata and Complexity: Collected Papers. Westview Press, New York.

World Health Organization - WHO (2012). World Malaria Report 2012. WHO Press, Geneva. 